\title{
Stress and Corticosteroids Modulate Muscarinic Long Term Potentiation (mLTP) in the Hippocampus
}

\author{
Efrat Shavit Stein ${ }^{1}$, Ze'Ev Itsekson Hayosh $^{1}$, Andreas Vlachos ${ }^{2}$ and Nicola Maggio ${ }^{1,3,4,5 *}$ \\ ${ }^{1}$ Department of Neurology, The Chaim Sheba Medical Center at Tel Hashomer, Ramat Gan, Israel, ${ }^{2}$ Department of \\ Neuroanatomy, Institute of Anatomy and Cell Biology, Faculty of Medicine, University of Freiburg, Freiburg, Germany, \\ ${ }^{3}$ Department of Neurology and Neurosurgery, Sackler School of Medicine, Tel Aviv University, Tel Aviv, Israel, ${ }^{4}$ Sagol School \\ of Neuroscience, Tel Aviv University, Tel Aviv, Israel, ${ }^{5}$ Talpiot Medical Leadership Program, The Chaim Sheba Medical Center \\ at Tel Hashomer, Ramat Gan, Israel
}

Stress influences synaptic plasticity, learning and memory in a steroid hormone receptor dependent manner. Based on these findings it has been proposed that stress could be a major risk factor for the development of cognitive decline and dementia. Interestingly, evidence has been provided that stress also affects muscarinic, i.e., acetylcholine (ACh)mediated neurotransmission. To learn more about the impact of stress and steroids on synaptic plasticity, in this study, we investigated the effects of stress on muscarinic long term potentiation (mLTP). We report that multiple, unpredictable exposure to stress depresses carbachol $(0.5 \mu \mathrm{M})$-induced $\mathrm{mLTP}$, while this effect of stress is not observed in hippocampal slices prepared from mice exposed only to a single stressful procedure. Furthermore, we demonstrate that activation of distinct steroid hormone receptors

OPEN ACCESS

Edited by: Marco Martina, Northwestern University, United States

Reviewed by:

Clive R. Bramham,

University of Bergen, Norway Phillip R. Zoladz,

Ohio Northern University, United States

*Correspondence: Nicola Maggio nicola.maggio@sheba.health.gov.il

Received: 30 July 2017 Accepted: 08 September 2017 Published: 21 September 2017

Citation:

Shavit Stein E, Itsekson Hayosh Z, Vlachos $A$ and Maggio $N$ (2017) Stress and Corticosteroids Modulate Muscarinic Long Term Potentiation (mLTP) in the Hippocampus.

Front. Cell. Neurosci. 11:299. doi: 10.3389/fncel.2017.00299 is involved in stress-mediated alterations of mLTP. Activation of mineralocorticoid receptors (MR) promotes $\mathrm{mLTP}$, while glucocorticoid receptor (GR) activity impairs $\mathrm{mLTP}$. These effects of multiple unpredictable stress on $\mathrm{mLTP}$ are long-lasting since they are detected even two weeks after the last stressful experience. Thus, multiple unpredictable events rather than a single stressful experience affect $\mathrm{mLTP}$ in a steroid hormone receptor dependent manner, suggesting that chronic unpredictable stress can lead to lasting alterations in hippocampal cholinergic plasticity.

Keywords: stress, corticosteroids, muscarinic LTP, cholinergic transmission, Alzheimer's disease, hippocampus

\section{INTRODUCTION}

Stress has a major impact on synaptic plasticity, learning and memory (Joëls et al., 2008; Joëls and Baram, 2009). Exposure to repeated, unpredictable stress is a risk factor for neurological deficits in neurodegenerative diseases (Goldstein, 2011). Specifically, chronic stress has been shown to accelerate the onset and progression of cognitive decline in Alzheimer's disease (AD) patients (Simard et al., 2009; Solas et al., 2010; Dhikav and Anand, 2011). The mechanisms underlying this phenomenon are not completely understood, although evidence has been provided that stress may increase hippocampal neurodegeneration (Jeong et al., 2006) via tau hyperphosphorylation (Sotiropoulos et al., 2011) and A-beta pathology (Srivareerat et al., 2009).

Exposure of animals to various forms of stress has major effects on the ability of hippocampal neurons to express electrically induced (tetanic) long term potentiation (LTP) of excitatory synapses (Pavlides et al., 2002; Alfarez et al., 2003; Karst and Joëls, 2003; Maggio and Segal, 2007, 2011, 2012b; Krugers et al., 2010; Segev et al., 2014; Sharvit et al., 2015). 
Indeed, robust experimental evidence exists that corticosteroids mediate the effects of stress on synaptic plasticity (Pavlides et al., 1994, 1995, 1996; Krugers et al., 2005; Wiegert et al., 2005; Maggio and Segal, 2007, 2009a,b, 2012a; Joëls et al., 2009). Interestingly, stress has been also linked to alterations in acetylcholine (ACh)-mediated neurotransmission (Kaufer et al., 1998; Farchi et al., 2007), e.g., by mediating alternative splicing of the primary ACh-hydrolyzing enzyme ACh-esterase (Farchi et al., 2007). Considering: (1) the relevance of the ACh in cognitive functions (Levey, 1996; Hut and Van der Zee, 2011); (2) the fact that deficits in cholinergic neurotransmission have been reported in a number of neuropsychiatric diseases, including AD (Yan and Feng, 2004; Berson et al., 2012); and (3) our earlier work on corticosteroid receptor mediated modulation of tetanus-induced LTP (Maggio and Segal, 2007, 2009b, 2012b), it appeared well warranted to test whether stress interferes with ACh-mediated neural plasticity in a corticosteroid dependent manner. Specifically, we were interested in testing whether this effect of stress is seen under conditions of repeated, unpredictable stress rather than after a single stressful procedure only.

In this study, the mechanisms involved in stress-induced modulation of muscarinic long term potentiation (mLTP) were assessed. MLTP is a form of synaptic plasticity that is induced by exposing hippocampal slices to carbachol, a muscarinic ACh-analog (Markram and Segal, 1990; Auerbach and Segal, 1996). Indeed, multiple, unpredictable exposure of stress depresses hippocampal mLTP in a corticosteroidreceptor dependent manner. This effect is long-lasting, since it can be detected 2 weeks after exposure to stress. Thus, our findings reveal a new mechanism on how stress affects synaptic plasticity via modulation of ACh-mediated synaptic plasticity, which may be important in the context of stress-related cognitive decline and the development of dementia.

\section{MATERIALS AND METHODS}

\section{Mice and Stress Protocol}

The study was approved by the Sheba Medical Center Institutional Animal Care and Use Committee (protocol number 698/11) which adheres to the national law and NIH rules. Mice were maintained in a 12-h light/dark cycle with food and water available ad libitum. Three months old male C57BL/6 mice were exposed either to acute stress or to multiple stress between 9:00 AM and 11:00 AM. The multiple stress protocol consisted of a repeated, unpredictable stressful procedure including a 15-min forced swim stress in a bucket of water (FSS) on the first day, a $30 \mathrm{~min}$ stay on an elevated platform stress (EPS) on the second day and a $2 \mathrm{~h}$ restraint in a narrow tube restrain stress (RS) on the third day. Age- and time-matched animals were exposed to a single stressful procedure only, which either was FSS, EP or RS. Control animals were kept in different cages and similarly handled, however not exposed to any stressful procedure. Experimental assessment of mLTP was carried out 1 day after the exposure to the stressful procedure.

\section{Pharmacology and Drugs}

The mineralocorticoid receptors (MR) agonist aldosterone (100 $\mu \mathrm{g} \mathrm{mg/kg;} \mathrm{Sigma)} \mathrm{and} \mathrm{the} \mathrm{glucocorticoid} \mathrm{receptor} \mathrm{(GR)}$ agonist dexamethasone (100 $\mu \mathrm{g} / \mathrm{kg}$; Sigma) were injected intraperitoneally (i.p.) $1.5 \mathrm{~h}$ prior to slice preparation. The MR antagonist spironolactone (Sigma) and the GR antagonist RU38486 (mifepristone, Sigma) were injected i.p. at a concentration of $20 \mathrm{mg} / \mathrm{kg}, 1 \mathrm{~h}$ prior to stress exposure. Control animals were i.p. injected with vehicle only. Drugs were prepared according to the manufacturer instructions and stored at $-20^{\circ} \mathrm{C}$ (Maggio and Segal, 2012c; Maggio et al., 2017). For the in vitro experiments, drugs were superfused into the recording medium at given concentrations with special care to prevent changes in temperature, $\mathrm{pH}$, flow rate, or degree of oxygenation of the artificial cerebrospinal fluid (ACSF).

\section{Slice Procedures and Electrophysiology}

Extracellular recordings in acute slices prepared from dorsal hippocampus were performed as previously described (Maggio and Segal, 2007, 2012a). Following anesthesia with ketamine/xylazine (100 mg/kg and $10 \mathrm{mg} / \mathrm{kg}$, respectively), animals were rapidly decapitated and $400 \mu \mathrm{m}$ hippocampal slices were prepared using a vibroslicer (NVSLM1 vibroslice, World Precision Instruments, Serasota, FL, USA). Slices were incubated for $1.5 \mathrm{~h}$ in a humidified, carbogenated $\left(5 \% \mathrm{CO}_{2}\right.$ and $95 \% \mathrm{O}_{2}$ ) gas atmosphere at $33 \pm 1^{\circ} \mathrm{C}$ and were perfused with ACSF [containing (in mM) $124 \mathrm{NaCl}, 2 \mathrm{KCl}, 26 \mathrm{NaHCO}_{3}$, $1.24 \mathrm{KH}_{2} \mathrm{PO}_{4}, 2.5 \mathrm{CaCl}_{2}, 2 \mathrm{MgSO}_{4}$, and 10 glucose, $\mathrm{pH} 7.4$ ] in a standard interface chamber. Recordings were made with a glass pipette containing $0.75 \mathrm{M} \mathrm{NaCl}$ (4 MOhm) placed in the stratum radiatum of CA1 as described previously (Maggio and Segal, 2007). Input-output curves were acquired from each slice prior to experimental assessment. Responses were digitized at $5 \mathrm{kHz}$ and stored on a computer. Spike 2 software (Cambridge Electronic Design, Milton, Cambridge, England) was used for data acquisition. Data are reported as means \pm SEM. Where appropriate, statistical analysis was performed with analysis of variance (ANOVA) followed by post hoc Tukey's comparisons.

\section{RESULTS}

\section{Exposure to Stress Affects mLTP at Schaffer Collateral-CA1 Synapses}

Three months old male $\mathrm{C} 57 \mathrm{BL} / 6$ mice were exposed to the following stress protocols: (1) a multiple, unpredictable stressful procedure including a FSS on the first day, an EPS (EP) on the second day and RS on the third day; or (2) a single stressful procedure of either FSS, EP or RS (see, Figure 1). Age- and time-matched control animals were not exposed to any stressful procedure but otherwise handled similarly.

Hippocampal slices were prepared the day after the (last) exposure of stress. In order to test for the effects of the two stress protocols on mLTP of Schaffer collateral-CA1 synapses, $0.5 \mu \mathrm{M}$ carbachol was bath applied for $40 \mathrm{~min}$ after acquiring a 

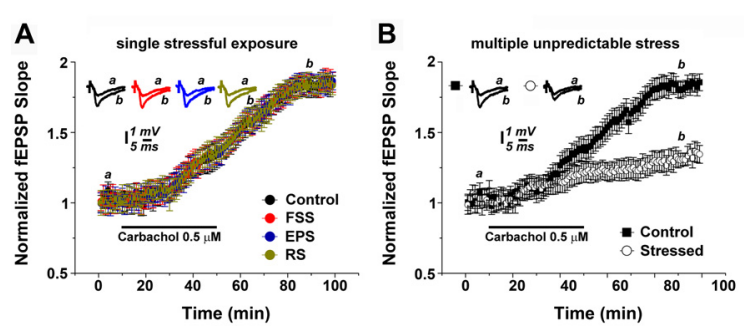

FIGURE 1 | Multiple unpredictable stressful events modulate muscarinic long term potentiation (mLTP). (A) Exposure of animals to a single stressful procedure does not affect $\mathrm{mLTP}$. (B) $\mathrm{mLTP}$ is depressed in hippocampal slices of animals which underwent multiple unpredictable stress exposure. Averaged field excitatory postsynaptic potentials (fEPSP) are plotted vs. time.

Representative traces at indicated times $(a, b)$ are shown on top of each section, $n=12$ slices for each experiment, refer to text for statistics. FSS, Forced swim stress; EPS, Elevated platform test; RS, Restrain stress.

10-min baseline of evoked field excitatory postsynaptic potentials (fEPSP). In slices of non-stressed control animals, carbachol reliably induced a slow increase in fEPSP slope (= mLTP), which continued to increase after the washout of carbachol, reaching its maximal value at about $80 \min (1.79 \pm 0.06)$. The plateau level was maintained till the end of the recordings (Figure 1A).

In hippocampal slices prepared from animals 1 day after a single stressful procedure, i.e., either after FSS, EP or RS, mLTP was not affected in comparison to controls: both the dynamics and magnitude of mLTP were indistinguishable between the groups (Figure 1A; $n=12$ slices per each group; one-way ANOVA; $p=0.67, F=0.50$, post hoc Tukey's; Figure 1A).

However, exposing animals to the multiple stress protocol had a major effect on the ability to express mLTP (Figure 1B). Specifically, at $80 \mathrm{~min}$ the fEPSP slope values were $1.29 \pm 0.07$ for the stressed animals compared to $1.81 \pm 0.07$ for the control animals $(n=12$ slices per each group; $p<0.001$; Figure 1B). We conclude from these experiments that multiple stressful experiences, rather than a single stressful event, alter cholinergic plasticity at Schaffer collateral-CA1 synapses.

\section{Corticosteroid Receptor Activation Modulates mLTP}

It is well-established that steroid hormone activation differentially modulates the ability of neurons to express electrically induced (tetanic) LTP of excitatory neurotransmission (McEwen, 1999; Joëls and Krugers, 2007; Joëls et al., 2008; Segal et al., 2010; Maggio and Segal, 2012b). Therefore, in a first step, we tested whether activation of MR or GR modulates mLTP-expression in non-stressed control animals (Figure 2A). GR or MR activation were achieved by single i.p. injections of aldosterone (aldo, $100 \mu \mathrm{g} / \mathrm{kg}$, an agonist of MR, i.e., MR activation) or dexamethasone (dexa, 100 $\mu \mathrm{g} / \mathrm{kg}$, an agonist of GR, i.e., GR activation) $1.5 \mathrm{~h}$ prior to slice preparations.

Figure 2A shows that MR or GR activation have distinct effects on the ability of neurons to express mLTP: while MR
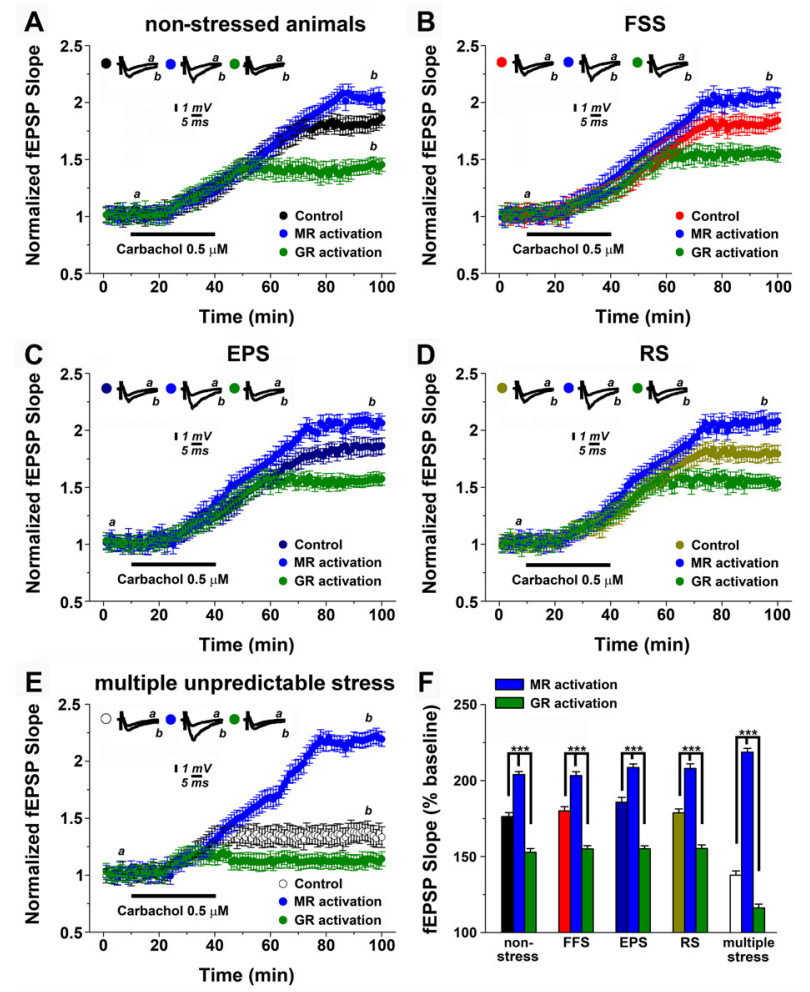

FIGURE 2 | Mineralocorticoid and glucocorticoid receptor (GR) mineralocorticoid receptors (MR, GR) modulation differentially affect $\mathrm{mLTP}$ after stress. (A) In non-stressed animals, MR and GR activation (respectively, single i.p. injection of the MR agonist aldosterone, $100 \mu \mathrm{g} / \mathrm{kg}$, i.e., MR activation or single i.p. injection of the GR agonist dexamethasone $100 \mu \mathrm{g} / \mathrm{kg}$, i.e., GR activation) enhances or depresses $\mathrm{mLTP}$, respectively. (B-D) A similar effect of MR and GR is observed upon a single stressful exposure. In these experiments, MR activation was obtained by blocking GR with mifepristone (20 mg/kg) prior to stress exposure, while GR activation was obtained by blocking MR with spironolactone $(20 \mathrm{mg} / \mathrm{kg}$ ) prior to stress exposure. (E) MR and GR inhibition results in stronger effects on either enhancement or depression of $\mathrm{mLTP}$, with GR inhibition preventing alterations in $\mathrm{mLTP}$ following multiple unpredictable stress. (F) Summary graph and combined analysis of experimental data. Further details of the results and the statistical comparisons are described in the text. Statistical analyses were made with two-ways analysis of variance (ANOVA), followed by post hoc Tukey's comparisons. FSS, Forced swim stress; EPS, Elevated platform test; RS, Restrain stress.

activation enhanced $\operatorname{mLTP}(2.02 \pm 0.049$ at $95 \mathrm{~min}$ of recordings compared to $1.80 \pm 0.072$ of controls), GR activation impaired $\operatorname{mLTP}(1.43 \pm 0.064 ; p<0.0001)$.

\section{Effects of Corticosteroid Receptor Activation on mLTP after Acute Stress}

We then tested for the role of MR and GR after exposure to a single stressful event. Previous work disclosed that blocking GR with mifepristone $(20 \mathrm{mg} / \mathrm{kg})$ under conditions of stress has similar effects to MR activation in non-stressed animals, while spironolactone $(20 \mathrm{mg} / \mathrm{kg})$ a blocker of MR results in GR activation under stress conditions (Avital et al., 2006). Drugs were injected $1 \mathrm{~h}$ prior to the stressful experience, i.e., FSS (Figure 2B), EPS (Figure 2C) or RS (Figure 2D). 
Indeed, GR-inhibition under stress conditions, i.e., MR activation improved mLTP, while MR inhibition under stress conditions, i.e., GR activation had the opposite effect (see, Figures 2A-D). The differential effects of steroid hormone activation did not differ from those occurring in the non-stressed animals (two-way ANOVA for multiple comparisons among means, $F=128.4, p<0.001$, Figure 2F). These experiments confirm and extend the role of MR and GR activation on mLTP modulation and provide additional support for the notion that a single stressful exposure to stress does not modulate the ability of neurons to express hippocampal cholinergic plasticity.

\section{Inhibition of GR Prevents Alterations in mLTP Under Conditions of Multiple, Unpredictable Stress}

What is the effect of corticosteroid receptors under conditions of multiple, unpredictable stress? In this set of experiments, animals were injected with either mifepristone or spironolactone $1 \mathrm{~h}$ prior each stressful experience (Figure 2E). Similar to the results described above, under stress conditions GR-inhibition, i.e., MR activation improved mLTP, while MR-inhibition, that is GR activation had a detrimental effect on the ability of neurons to express $\operatorname{mLTP}(F=226.2$, $p<0.001)$. However, GR-inhibition in fact restored mLTP back to levels seen under non-stressed conditions, or in animals that received only one stressful procedure (Figure $2 \mathrm{E}$ ). In contrast MR-inhibition almost completely blocked the ability of neurons to express mLTP ( $F_{\text {interaction between factors }}=25.5$, $p<0.001$; Figure 2F). We conclude from these results that multiple stressful exposures to stress impair the ability of neurons to express mLTP while activation of MR and GR has a more profound effect on mLTP with GR-inhibition restoring the ability of neurons to express mLTP.

\section{In Vitro Effects of Corticosteroid Receptor Activation on mLTP}

In order to confirm and extend the above findings, which suggest that stress affects hippocampal cholinergic plasticity via corticosteroid receptor activation, we next performed in vitro pharmacological experiments in hippocampal slices prepared from non-stressed control animals. In these experiments mifepristone $(500 \mathrm{nM})$ and corticosterone $(100 \mathrm{nM})$ was used to activate $\mathrm{MR}$, or spironolactone $(500 \mathrm{nM})$ and corticosterone (100 nM) to achieve GR activation (Maggio and Segal, 2007). As shown in Figure 3, MR activation resulted in a $1.99 \pm 0.091$ (at $100 \mathrm{~min}$ of recordings) mLTP, while GR stimulation resulted in $1.29 \pm 0.075$ of muscarinic potentiation (Figure 3A). One-way ANOVA comparing the results in control slices and those obtained in slices where MR and GR were stimulated, revealed significant differences among groups $(F=17.2, p<0.0001$, Figure 3B). Hence, similar to our in vivo pharmacological experiments this set of experiments disclosed the differential effect of MR and GR on mLTP.
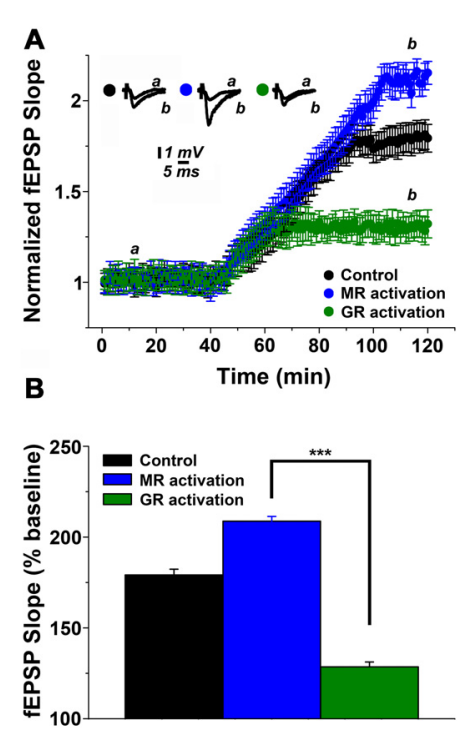

FIGURE 3 | Steroid hormone receptor mediated modulation of $\mathrm{mLTP}$ in hippocampal slices. (A) MR activation enhances while GR activation depresses $\mathrm{mLTP}$. In these experiments mifepristone $(500 \mathrm{nM})$ and corticosterone (100 nM) were used to activate MR while spironolactone $(500 \mathrm{nM})$ and corticosterone $(100 \mathrm{nM})$ were used to achieve GR-activation. (B) Summary graph and combined analysis of experimental data. Further details of the results and the statistical comparisons are described in the text. Statistical analyses were made with one-way ANOVA, followed by post hoc Tukey's comparisons. ${ }^{* * *} p<0.0001$.

\section{GR Activation Modulates $\mathrm{N}$-methyl-D-aspartate Receptor (NMDAR) Activity}

It has been previously shown that mLTP results from the cholinergic activation of $\mathrm{N}$-methyl-D-aspartate receptors (NMDARs), which leads to enhanced synaptic responses following exposure of hippocampal slices to $0.5 \mu \mathrm{M}$ carbachol (Markram and Segal, 1990; Auerbach and Segal, 1996). Therefore, we predicted that GR-activity depresses mLTP by reducing NMDAR-evoked potentials. In order to test this hypothesis, we recorded evoked NMDAR potentials in presence of $0 \mu \mathrm{M} \mathrm{Mg}^{2+}, 10 \mu \mathrm{M}$ DNQX and $10 \mu \mathrm{M}$ Glycine, as previously described (Maggio and Segal, 2007). Indeed, in this setting, exposure to $100 \mathrm{nM}$ dexamethasone resulted in a depression of NMDAR-mediated potentials (Figure 4). We conclude that GR activation may act in our experimental setting by modulating NMDARs.

\section{Multiple, Unpredictable Stress Persistently Impairs MLTP}

Finally, we wondered whether the effects of multiple, unpredictable stress on mLTP are transient or long-lasting. Thus, we exposed animals to the multiple stressful procedure and performed hippocampal slices 2 weeks later to test for the ability to induce mLTP (Figure 5). Strikingly, in these experiments $0.5 \mu \mathrm{M}$ carbachol resulted still in a lower magnitude mLTP compared to non-stressed animals $(1.45 \pm 0.06$ and $1.81 \pm 0.09$, respectively, $p<0.001)$. 


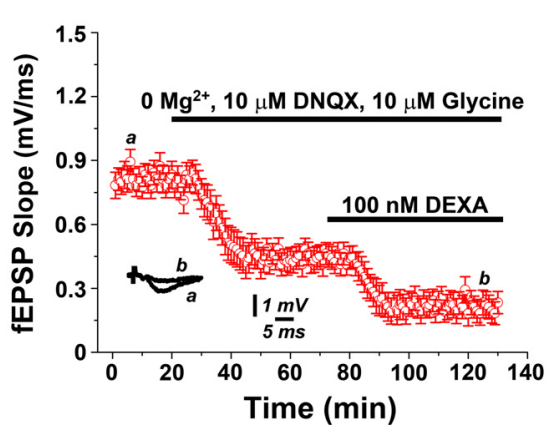

FIGURE 4 | GR activation depresses N-methyl-D-aspartate receptor (NMDAR)-mediated potentials. NMDAR-mediated field potentials recorded from Schaffer collateral-CA1 synapses in presence of $0 \mu \mathrm{M} \mathrm{Mg}^{2+}, 10 \mu \mathrm{M}$ DNQX and $10 \mu \mathrm{M}$ Glycine are depressed upon exposure to the GR agonist dexamethasone $(100 \mathrm{nM})$.

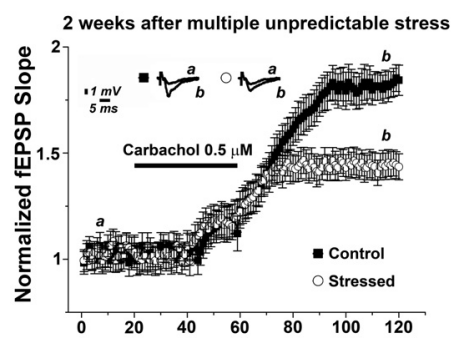

FIGURE 5 | Multiple stressful experiences persistently impair mLTP. mLTP evoked by $0.5 \mu \mathrm{M}$ carbachol in animals exposed to multiple stressful experiences 2 weeks prior slice preparation is significantly reduced compared to the $\mathrm{mLTP}$ evoked in age- and time-matched control, non-stressed animals. Averaged fEPSP are plotted vs. time. Representative traces at indicated times $(\mathrm{a}, \mathrm{b})$ are shown on top of each section, $n=12$ slices for each experiment.

These results show that the effects of multiple, unpredictable stress are not only seen after a few hours following the last exposure to stress. We conclude that multiple stressful events can have long-lasting effects on hippocampal cholinergic plasticity.

\section{DISCUSSION}

In this study, we addressed the role of stress in modulating mLTP. We found that a single exposure to stress does not influence mLTP, while multiple stressful procedures reduce the magnitude of mLTP. This effect on mLTP is long-lasting, since it can be detected even two weeks following the stressful events. Corticosteroids are involved in mediating the effect of multiple, unpredictable stress on mLTP. Inhibition of GR prevents alterations in mLTP, while GR activity blocks mLTP likely via a reduction in NMDA-R currents. Considering the relevance of ACh-mediated synaptic plasticity under physiological and pathological conditions (Markram and Segal, 1990; Auerbach and Segal, 1996; Hut and Van der Zee, 2011), these results provide new important insight on how chronic unpredictable stress may contribute to the development of cognitive decline and dementia.

It has been previously reported that repeated stress predisposes to the onset of dementia and $\mathrm{AD}$. The mechanisms underlying this phenomenon are not completely understood (Simard et al., 2009; Solas et al., 2010; Millan et al., 2012). The results of the present study reveal that stress directly affects cholinergic plasticity and may thus impair ACh-mediated learning and memory (Hut and Van der Zee, 2011). Alterations of cholinergic transmission are considered a first, crucial step for the onset of Alzheimer's dementia (Millan et al., 2012). As such stress may contribute to the pathophysiology of the disease from its early stages. If this is indeed the case, stress management should be timely adopted in order to counteract chronic stress and therefore prevent (or at least postpone) the onset of the disease.

The mechanisms through which chronic stress modulates hippocampal cholinergic plasticity warrant further investigation. In this study, we were able to provide first evidence that corticosteroids are involved in stress-mediated alterations of hippocampal mLTP. While MR activation promotes mLTP, GR activation impairs the ability of neurons to express mLTP. Yet, the precise molecular mechanisms through which these differential effects of corticosteroid receptors are mediated are unknown. It is interesting to speculate though that they may resemble effects described in the context of tetanusinduced synaptic plasticity (Joëls and Krugers, 2007; Maggio and Segal, 2012b). Consistent with the results of the present study, it has been previously shown that GR activation impairs LTP by depressing NMDAR potentials (Maggio and Segal, 2007). Conversely, MR activation promotes LTP through the activation of voltage-gated calcium channels (VGCCs; Joëls and Krugers, 2007; Groeneweg et al., 2012; Maggio and Segal, 2012a). Since, it has been reported that muscarinic M1 receptor can enhance synaptic plasticity through VGCCs (Giessel and Sabatini, 2010), it is conceivable that the positive effects of MR on mLTP may involve this pathway. Nevertheless, additional experiments are needed in order to clarify this hypothesis.

Another interesting observation of the present study concerns differences between a single stressful event and multiple, unpredictable stressful procedures. Previous work has demonstrated that exposure to a single stressful event affects tetanic stimulation induced LTP (Maggio and Segal, 2007). Unexpectedly, this is not the case for mLTP. A possible explanation is that mLTP does not only depend on NMDARs. Hence, additional mechanisms may compensate for GR mediated changes in NMDARs under conditions of single stressful events, e.g., changes in VGCCs. Under conditions of chronic stress these compensatory mechanisms may fail or could even be directly or indirectly affected by multiple, unpredictable stress. In order to better understand the effects of stress on cholinergic plasticity it will be important to assess compensatory mechanisms and the ability of neurons to express homeostatic plasticity. It is for example conceivable that different compensatory mechanisms may also act at different points in time following acute or 
chronic stress. For example, in this study, we performed slices 1 day after the exposure to single stress, while in previous studies we assessed tetanic LTP $1 \mathrm{~h}$ upon stress exposure. Hence, in the current conditions, synapses may recover from the stressful experience and as such exhibit a normal NMDAR-dependent mLTP. This theory is supported by our findings in juvenile animals where a normal stimulusinduced LTP could be evoked 1 day after stress in the dorsal hippocampus (Maggio and Segal, 2011). As the case may be, additional work is needed in order to better understand the time-course of alterations in mLTP and putative compensatory mechanisms.

Yet, the effects of multiple stress exposure on mLTP resemble those reported on tetanus-induced LTP. Specifically, we (Maggio and Segal, 2011) and others (Pavlides et al., 2002; Alfarez et al., 2003; Karst and Joëls, 2003; Krugers et al., 2010) have shown that chronic, multiple exposure to stress impairs tetanus-induced LTP. Therefore it is tempting to speculate that

\section{REFERENCES}

Albrecht, A., Müller, I., Ardi, Z., Çaliskan, G., Gruber, D., Ivens, S., et al. (2017). Neurobiological consequences of juvenile stress: a GABAergic perspective on risk and resilience. Neurosci. Biobehav. Rev. 74, 21-43. doi: 10.1016/j.neubiorev. 2017.01.005

Alfarez, D. N., Joëls, M., and Krugers, H. J. (2003). Chronic unpredictable stress impairs long-term potentiation in rat hippocampal CA1 area and dentate gyrus in vitro. Eur. J. Neurosci. 17, 1928-1934. doi: 10.1046/j.1460-9568.2003. 02622.x

Auerbach, J. M., and Segal, M. (1996). Muscarinic receptors mediating depression and long-term potentiation in rat hippocampus. J. Physiol. 492, 479-493. doi: 10.1113/jphysiol.1996.sp021323

Avital, A., Segal, M., and Richter-Levin, G. (2006). Contrasting roles of corticosteroid receptors in hippocampal plasticity. J. Neurosci. 26, 9130-9134. doi: 10.1523/JNEUROSCI.1628-06.2006

Berson, A., Barbash, S., Shaltiel, G., Goll, Y., Hanin, G., Greenberg, D. S., et al. (2012). Cholinergic-associated loss of hnRNP-A/B in Alzheimer's disease impairs cortical splicing and cognitive function in mice. EMBO Mol. Med. 4, 730-742. doi: 10.1002/emmm.201100995

Dhikav, V., and Anand, K. (2011). Potential predictors of hippocampal atrophy in Alzheimer's disease. Drugs Aging 28, 1-11. doi: 10.2165/11586390-00000000000000

Farchi, N., Shoham, S., Hochner, B., and Soreq, H. (2007). Impaired hippocampal plasticity and errors in cognitive performance in mice with maladaptive AChE splice site selection. Eur. J. Neurosci. 25, 87-98. doi: 10.1111/j.1460-9568.2006. 05249.x

Giessel, A. J., and Sabatini, B. L. (2010). M1 muscarinic receptors boost synaptic potentials and calcium influx in dendritic spines by inhibiting postsynaptic SK channels. Neuron 68, 936-947. doi: 10.1016/j.neuron.2010.09.004

Goldstein, D. S. (2011). Stress, allostatic load, catecholamines, and other neurotransmitters in neurodegenerative diseases. Endocr. Regul. 45, 91-98. doi: 10.4149/endo_2011_0_91

Groeneweg, F. L., Karst, H., de Kloet, E. R., and Joëls, M. (2012). Mineralocorticoid and glucocorticoid receptors at the neuronal membrane, regulators of nongenomic corticosteroid signalling. Mol. Cell. Endocrinol. 350, 299-309. doi: $10.1016 /$ j.mce.2011.06.020

Hut, R. A., and Van der Zee, E. A. (2011). The cholinergic system, circadian rhythmicity, and time memory. Behav. Brain Res. 221, 466-480. doi: 10.1016/j. bbr.2010.11.039

Jeong, Y. H., Park, C. H., Yoo, J., Shin, K. Y., Ahn, S. M., Kim, H. S., et al. (2006). Chronic stress accelerates learning and memory impairments and increases amyloid deposition in APPV717I-CT100 transgenic mice, an Alzheimer's disease model. FASEB J. 20, 729-731. doi: 10.1096/fj.05-4265fje multiple stress exposure may reduce the ability of neurons to express NMDAR-dependent synaptic plasticity, regardless of the stimulus used to induce/probe plasticity thus providing a cellular substrate for the development of both hippocampal-dependent cognitive (Sotiropoulos et al., 2011) and emotional (Albrecht et al., 2017) deficits after a prolonged exposure to stressful events.

\section{AUTHOR CONTRIBUTIONS}

AV and NM: designed the study and wrote the article. ESS, ZIH and NM: performed and analyzed experiments.

\section{ACKNOWLEDGMENTS}

This research was supported by a Grant from the German-Israeli Foundation for Scientific Research and Development (GIF 2290$2242.1 / 2011$ to NM).

Joëls, M., and Baram, T. Z. (2009). The neuro-symphony of stress. Nat. Rev. Neurosci. 10, 459-466. doi: 10.1038/nrn2632

Joëls, M., and Krugers, H. J. (2007). LTP after stress: up or down? Neural Plast. 2007:93202. doi: 10.1155/2007/93202

Joëls, M., Krugers, H., and Karst, H. (2008). Stress-induced changes in hippocampal function. Prog. Brain Res. 167, 3-15. doi: 10.1016/s00796123(07)67001-0

Joëls, M., Krugers, H. J., Lucassen, P. J., and Karst, H. (2009). Corticosteroid effects on cellular physiology of limbic cells. Brain Res. 1293, 91-100. doi: 10.1016/j. brainres.2009.03.036

Karst, H., and Joëls, M. (2003). Effect of chronic stress on synaptic currents in rat hippocampal dentate gyrus neurons. J. Neurophysiol. 89, 625-633. doi: $10.1152 /$ jn.00691.2002

Kaufer, D., Friedman, A., Seidman, S., and Soreq, H. (1998). Acute stress facilitates long-lasting changes in cholinergic gene expression. Nature 393, 373-377. doi: $10.1038 / 30741$

Krugers, H. J., Alfarez, D. N., Karst, H., Parashkouhi, K., van Gemert, N., and Joels, M. (2005). Corticosterone shifts different forms of synaptic potentiation in opposite directions. Hippocampus 15, 697-703. doi: 10.1002/hipo. 20092

Krugers, H. J., Lucassen, P. J., Karst, H., and Joels, M. (2010). Chronic stress effects on hippocampal structure and synaptic function: relevance for depression and normalization by anti-glucocorticoid treatment. Front. Synaptic Neurosci. 2:24. doi: 10.3389/fnsyn.2010.00024

Levey, A. I. (1996). Muscarinic acetylcholine receptor expression in memory circuits: implications for treatment of Alzheimer disease. Proc. Natl. Acad. Sci. U S A 93, 13541-13546. doi: 10.1073/pnas.93.24.13541

Maggio, N., and Segal, M. (2007). Striking variations in corticosteroid modulation of long-term potentiation along the septotemporal axis of the hippocampus. J. Neurosci. 27, 5757-5765. doi: 10.1523/JNEUROSCI.015507.2007

Maggio, N., and Segal, M. (2009a). Differential corticosteroid modulation of inhibitory synaptic currents in the dorsal and ventral hippocampus. J. Neurosci. 29, 2857-2866. doi: 10.1523/JNEUROSCI.4399-08.2009

Maggio, N., and Segal, M. (2009b). Differential modulation of long-term depression by acute stress in the rat dorsal and ventral hippocampus. J. Neurosci. 29, 8633-8638. doi: 10.1523/JNEUROSCI.190109.2009

Maggio, N., and Segal, M. (2011). Persistent changes in ability to express long-term potentiation/depression in the rat hippocampus after juvenile/adult stress. Biol. Psychiatry 69, 748-753. doi: 10.1016/j.biopsych.2010.11.026

Maggio, N., and Segal, M. (2012a). Cellular basis of a rapid effect of mineralocorticosteroid receptors activation on LTP in ventral hippocampal slices. Hippocampus 22, 267-275. doi: 10.1002/hipo.20893 
Maggio, N., and Segal, M. (2012b). Steroid modulation of hippocampal plasticity: switching between cognitive and emotional memories. Front. Cell. Neurosci. 6:12. doi: $10.3389 /$ fncel.2012.00012

Maggio, N., and Segal, M. (2012c). Stress and corticosteroid modulation of seizures and synaptic inhibition in the hippocampus. Exp. Neurol. 234, 200-207. doi: 10.1016/j.expneurol.2011.12.035

Maggio, N., Shavit Stein, E., and Segal, M. (2017). Complex modulation by stress of the effect of seizures on long term potentiation in mouse hippocampal slices. Hippocampus 27, 860-870. doi: 10.1002/hipo.22736

Markram, H., and Segal, M. (1990). Long-lasting facilitation of excitatory postsynaptic potentials in the rat hippocampus by acetylcholine. J. Physiol. 427, 381-393. doi: 10.1113/jphysiol.1990.sp018177

McEwen, B. S. (1999). Stress and hippocampal plasticity. Annu. Rev. Neurosci. 22, 105-122. doi: 10.1146/annurev.neuro.22.1.105

Millan, M. J., Agid, Y., Brüne, M., Bullmore, E. T., Carter, C. S., Clayton, N. S., et al. (2012). Cognitive dysfunction in psychiatric disorders: characteristics, causes and the quest for improved therapy. Nat. Rev. Drug Discov. 11, 141-168. doi: $10.1038 / \operatorname{nrd} 3628$

Pavlides, C., Kimura, A., Magariños, A. M., and McEwen, B. S. (1994). Type I adrenal steroid receptors prolong hippocampal long-term potentiation. Neuroreport 5, 2673-2677. doi: 10.1097/00001756-199412000-00067

Pavlides, C., Nivon, L. G., and McEwen, B. S. (2002). Effects of chronic stress on hippocampal long-term potentiation. Hippocampus 12, 245-257. doi: 10.1002/hipo.1116

Pavlides, C., Ogawa, S., Kimura, A., and McEwen, B. S. (1996). Role of adrenal steroid mineralocorticoid and glucocorticoid receptors in long-term potentiation in the CA1 field of hippocampal slices. Brain Res. 738, 229-235. doi: 10.1016/s0006-8993(96)00776-7

Pavlides, C., Watanabe, Y., Magariños, A. M., and McEwen, B. S. (1995). Opposing roles of type I and type II adrenal steroid receptors in hippocampal long-term potentiation. Neuroscience 68, 387-394. doi: 10.1016/0306-4522(95)00151-8

Segal, M., Richter-Levin, G., and Maggio, N. (2010). Stress-induced dynamic routing of hippocampal connectivity: a hypothesis. Hippocampus 20, 1332-1338. doi: 10.1002/hipo.20751

Segev, A., Rubin, A. S., Abush, H., Richter-Levin, G., and Akirav, I. (2014). Cannabinoid receptor activation prevents the effects of chronic mild stress on emotional learning and LTP in a rat model of depression. Neuropsychopharmacology 39, 919-933. doi: 10.1038/npp.2013.292
Sharvit, A., Segal, M., Kehat, O., Stork, O., and Richter-Levin, G. (2015). Differential modulation of synaptic plasticity and local circuit activity in the dentate gyrus and CA1 regions of the rat hippocampus by corticosterone. Stress 18, 319-327. doi: 10.3109/10253890.2015.1023789

Simard, M., Hudon, C., and van Reekum, R. (2009). Psychological distress and risk for dementia. Curr. Psychiatry Rep. 11, 41-47. doi: 10.1007/s11920-009 $-0007-\mathrm{Z}$

Solas, M., Aisa, B., Mugueta, M. C., Del Rio, J., Tordera, R. M., and Ramirez, M. J. (2010). Interactions between age, stress and insulin on cognition: implications for Alzheimer's disease. Neuropsychopharmacology 35 , 1664-1673. doi: 10.1038/npp.2010.13

Sotiropoulos, I., Catania, C., Pinto, L. G., Silva, R., Pollerberg, G. E., Takashima, A., et al. (2011). Stress acts cumulatively to precipitate Alzheimer's disease-like tau pathology and cognitive deficits. J. Neurosci. 31, 7840-7847. doi: 10.1523/JNEUROSCI.0730-11.2011

Srivareerat, M., Tran, T. T., Alzoubi, K. H., and Alkadhi, K. A. (2009). Chronic psychosocial stress exacerbates impairment of cognition and long-term potentiation in $\beta$-amyloid rat model of Alzheimer's disease. Biol. Psychiatry 65 , 918-926. doi: 10.1016/j.biopsych.2008.08.021

Wiegert, O., Pu, Z., Shor, S., Joëls, M., and Krugers, H. (2005). Glucocorticoid receptor activation selectively hampers $N$-methyl- $D$-aspartate receptor dependent hippocampal synaptic plasticity in vitro. Neuroscience 135, 403-411. doi: 10.1016/j.neuroscience.2005.05.039

Yan, Z., and Feng, J. (2004). Alzheimer's disease: interactions between cholinergic functions and $\beta$-amyloid. Curr. Alzheimer Res. 1, 241-248. doi: $10.2174 / 1567205043331992$

Conflict of Interest Statement: The authors declare that the research was conducted in the absence of any commercial or financial relationships that could be construed as a potential conflict of interest.

Copyright (c) 2017 Shavit Stein, Itsekson Hayosh, Vlachos and Maggio. This is an open-access article distributed under the terms of the Creative Commons Attribution License (CC BY). The use, distribution or reproduction in other forums is permitted, provided the original author(s) or licensor are credited and that the original publication in this journal is cited, in accordance with accepted academic practice. No use, distribution or reproduction is permitted which does not comply with these terms. 